\title{
Generalization of the Plana Formula
}

\author{
V. I. Kuzovatov ${ }^{1 *}$ \\ ${ }^{1}$ Siberian Federal University \\ pr. Svobodnyi 79, Krasnoyarsk, 660041 Russia
}

Received March 9, 2017

\begin{abstract}
We obtain an analog of the Plana formula, which is essential in obtaining the functional equation for the classical Riemann zeta-function.
\end{abstract}

DOI: $10.3103 / \mathrm{S} 1066369 \mathrm{X} 18050067$

Keywords: Plana formula, entire function, integral representation.

\section{INTRODUCTION}

The aim of the paper is obtaining an analog of the Plana formula (see, e.g., [1], Chap. 7, exercise 7) which is essential for finding the functional equation (see, e.g., [2], Chap. 2, section 9) for the classical Riemann zeta-function. Kuzovatov and A. M. Kytmanov [3] obtained an analog of the Plana formula under more strong restrictions on rational function. In the paper we remove these restrictions.

Let $\varphi$ be a holomorphic function, bounded for all $z$ such that $x_{1} \leq \operatorname{Re} z \leq x_{2} ; x_{1}, x_{2}$ are integers. The classical Plana formula connects the sum of values of $\varphi(z)$ at integer points with some integrals:

$$
\begin{aligned}
\frac{1}{2} \varphi\left(x_{1}\right)+\varphi & \left(x_{1}+1\right)+\varphi\left(x_{1}+2\right)+\cdots+\varphi\left(x_{2}-1\right)+\frac{1}{2} \varphi\left(x_{2}\right) \\
= & \int_{x_{1}}^{x_{2}} \varphi(z) d z+\frac{1}{i} \int_{0}^{\infty} \frac{\varphi\left(x_{2}+i y\right)-\varphi\left(x_{2}-i y\right)+\varphi\left(x_{1}-i y\right)-\varphi\left(x_{1}+i y\right)}{e^{2 \pi y}-1} d y .
\end{aligned}
$$

Concerning generalizations of the zeta-function, we note that in 1950s Gelfand, Levitan, and Dikii (see, e.g., [4-6]) studied the zeta-function associated to eigenvalues of the Sturm-Lioville operator. As it turned out, its value is connected with the trace of the operator. Further their approach was developed by Lidskii and Sadovnichii [7] who considered a class of entire functions of one variable, defined the zeta-function of their zeroes and investigated its domain of analytic continuation. Smagin and Shubin [8] constructed the zeta-functions for elliptic operators, as long for operators of more general type, proved a possibility of meromorphic continuation of the zeta-function and gave some information on its poles.

Multidimensional results were obtained by A. M. Kytmanov and Myslivetz [9]. They introduced the concept of zeta-function associated with a system of meromorphic functions $f=\left(f_{1}, \ldots, f_{n}\right)$ in $\mathbb{C}^{n}$. With the help of the residue theory, an integral representation for the zeta-function was given but under fulfillment of some strong conditions on the system $f_{1}, \ldots, f_{n}$.

In [10], with the help of the residue theory, Kuzovatov and A. A. Kytmanov obtained two integral representation for zeta-function constructed by zeroes of an entire function of finite order on the complex plane. With the help of these representations, they described a domain which the zeta-functions can be extended to.

\footnotetext{
${ }^{*}$ E-mail: kuzovatov@yandex.ru.
} 


\section{AUXILIARY RESULTS}

Let us formulate the results from [10]. Let $f(z)$ be an entire function of order $\rho$ in $\mathbb{C}$. Consider the equation

$$
f(z)=0 .
$$

Denote by $N_{f}=f^{-1}(0)$ the set of all solutions to (2) (we take every zero as many times as its multiplicity). The numbers of roots is at most countable.

The zeta-function $\zeta_{f}(s)$ of roots of Eq. $(2)$ is defined in the following way:

$$
\zeta_{f}(s)=\sum_{a \in N_{f}}(-a)^{-s}
$$

where $s \in \mathbb{C}$. The minus sign in the definition is taken for convenient recording of the integrals below.

Now we will give an integral representation for the zeta-function $\zeta_{f}(s)$ of zeroes $z_{n}$ of $f$ which are

$$
z_{n}=-q_{n}+i s_{n}, \quad q_{n}>0 .
$$

Let us denote

$$
F(f, x)=\sum_{n=1}^{\infty} e^{z_{n} x} .
$$

We will assume that $\operatorname{Re} s=\sigma>1$ and the following conditions hold:

$$
\begin{gathered}
\qquad \underline{\varliminf_{n \rightarrow \infty}} \frac{q_{n}}{n}>0, \\
\text { the series } \sum_{n=1}^{\infty}\left(\frac{1}{q_{n}}\right)^{\sigma-1} \text { converges. }
\end{gathered}
$$
[10]

For investigation of convergence of (3) we will use the Cauchy convergence test. For real $x$ we have

$$
\left|e^{z_{n} x}\right|=\left|e^{\left(-q_{n}+i s_{n}\right) x}\right|=\left|e^{-q_{n} x} \cdot e^{i s_{n} x}\right|=e^{-q_{n} x} .
$$

Therefore, making use of (4), we see that (3) converges if and only if

$$
\varlimsup_{n \rightarrow \infty} \sqrt[n]{e^{-q_{n} x}}=\varlimsup_{n \rightarrow \infty} e^{-\frac{q_{n} x}{n}}=\varlimsup_{n \rightarrow \infty} \frac{1}{e \frac{q_{n} x}{n}}<1,
$$

i.e., for $x>0$.

Theorem 1 ([10]). Let (4) and (5) hold, and $\operatorname{Re} s>1$. Then

$$
\zeta_{f}(s)=\frac{1}{\Gamma(s)} \int_{0}^{\infty} x^{s-1} F(f, x) d x
$$

where $F(f, x)$ is defined by $(3)$.

In the paper we will assume that $z_{n}=-q_{n}, q_{n}>0$, where $q_{n}$ is a sequence of naturals.

Consider the function

$$
F(f, 2 \pi i z)=\sum_{n=1}^{\infty} e^{z_{n} 2 \pi i z}=\sum_{n=1}^{\infty} e^{-q_{n} 2 \pi i z}
$$

For $z=x+i y$ we have

$$
\left|e^{-q_{n} 2 \pi i z}\right|=\left|e^{-q_{n} 2 \pi i(x+i y)}\right|=\left|e^{-q_{n} 2 \pi i x} \cdot e^{q_{n} 2 \pi y}\right|=e^{q_{n} 2 \pi y} .
$$


Therefore, the domain of convergence for series (6) is the set defined by

$$
\varlimsup_{n \rightarrow \infty} \sqrt[n]{e^{q_{n} 2 \pi y}}=\varlimsup_{n \rightarrow \infty} e^{\frac{q_{n} 2 \pi y}{n}}<1
$$

due to (4) it is equivalent to the inequality $y<0$, .

With the help of the change of variable $e^{-2 \pi i z}=w$, series $(6)$ is reduced to the form $\sum_{n=1}^{\infty} w^{q_{n}}$ or

$$
G(w)=\sum_{n=1}^{\infty} f_{n} w^{n}
$$

where

$$
f_{n}=\left\{\begin{array}{ll}
1, & n=q_{k} ; \\
0, & n \neq q_{k},
\end{array} \text { therefore, } \quad \varlimsup_{n \rightarrow \infty} \sqrt[n]{\left|f_{n}\right|}=1\right.
$$

We should note that an infinite number of coefficients $f_{n}$ in $(7)$ is distinct from zero.

The function $G(w)$ is unbounded as $|w| \rightarrow 1-0$, but it is holomorphic in the unit disk. Therefore, $G(w)$ could not be extended to the point 1. From the Fabry gap theorem (see, e.g., [11], \$ 2.3) it follows that we can take the coefficients of the series in such a way that the whole circle will be the natural boundary for $G(w)$.

Further we restrict ourselves by considering classes of rational functions $G(w)$ satisfying $(7)$. We recall

Theorem 2 (Szegö, [11], §6.1). Let

$$
G(w)=\sum_{n=0}^{\infty} f_{n} w^{n}
$$

be a power series and its coefficients $f_{n}$ take only a finite number of distinct values. Then either $G$ is a rational function or it could not be extended outside the unit disk.

In the case when the sum of (8) is a rational function, we have

$$
G(w)=\frac{P(w)}{1-w^{N}}
$$

where $P(w)$ is a polynomial, and $N$ is a natural.

By the theorem, $G(w)$ can have singularities (simple poles) only at the points

$$
w_{k}=e^{i \frac{2 \pi}{N} k}, \quad k=0,1, \ldots, N-1, \quad N \in \mathbb{N} .
$$

In terms of the $z$-variable, the singularities of $F(f, 2 \pi i z)$ are

$$
e^{-2 \pi i z}=w_{k}, \quad-2 \pi i z=i\left(\frac{2 \pi}{N} k+2 \pi l\right), \quad z=-\left(\frac{k}{N}+l\right), \quad l=0, \pm 1, \pm 2, \ldots,
$$

or, what is the same,

$$
z_{k, l}=l-\frac{k}{N}, \quad l=0, \pm 1, \pm 2, \ldots, \quad k=0,1, \ldots, N-1 .
$$

Let $q_{n}$ satisfy inequality (4). Assume additionally that $\operatorname{deg} P(w)=N$, i.e.,

$$
P(w)=a_{1} w+a_{2} w^{2}+\cdots+a_{N-1} w^{N-1}+w^{N}
$$

where, because of (7), the coefficients $a_{j} \in\{0,1\}, j=1, \ldots, N-1$.

We should note that if $\operatorname{deg} P(w)>N$, then in the expansion of $G(w)$ we can separate a part which contains only a finite number of terms; these terms do not influence on other ones with sufficiently large 
numbers. If $\operatorname{deg} P(w) \leq N$, then, in the expansion of $G(w)$, the coefficients of $P(w)$ occur; they are repeated periodically, because the expansion of $\left(1-w^{N}\right)^{-1}$ is a geometric series. We wrote separately the monomial $w^{N}$ for convenience of calculations.

We have an explicit expression for $F(f, 2 \pi i z)$ :

$$
F(f, 2 \pi i z)=\frac{P\left(e^{-2 \pi i z}\right)}{1-e^{-2 \pi i z N}} .
$$

We note that (9) gives analytic continuation of the functions $F(f, 2 \pi i z)$, introduced above and defined by (6). The domain of definition for (9) is the complex plane $\mathbb{C}$ except for the points $z_{k, l}$.

We note that in [3] the following conditions were required:

$$
1+F(f, 2 \pi i z)=-F(f,-2 \pi i z)
$$

and

$$
-(1+F(f,-2 \pi y))=F(f, 2 \pi y) .
$$

In terms of the rational function $G(w)$ the condition (10) is equivalent to the equality

$$
1+G(w)=-G\left(\frac{1}{w}\right) .
$$

In the present paper restrictions (10) and (11) are removed.

Further $\varphi(z)$ is a function holomorphic and bounded on the set $\left\{x_{1} \leq \operatorname{Re} z \leq x_{2}\right\}$; here $x_{1}$ and $x_{2}$ are integers.

Lemma 1. We have

$$
\lim _{y \rightarrow+\infty}\left(\varphi\left(x_{j}-i y\right) F(f, 2 \pi y)+\varphi\left(x_{j}+i y\right)[1+F(f,-2 \pi y)]\right)=0, \quad j=1,2 .
$$

Proof. We should note that $F(f, 2 \pi y)$ is obtained from the rational function $G(w)$ by substituting $w$ for $w=e^{-2 \pi y}$. Similarly, $F(f,-2 \pi y)$ is obtained from $G(w)$ by the change $w=e^{2 \pi y}$. Denoting $e^{2 \pi y}=t$, we write

$$
\begin{aligned}
F(f, 2 \pi y)=\frac{\frac{a_{1}}{t}+\frac{a_{2}}{t^{2}}+\cdots+\frac{a_{N-1}}{t^{N-1}}+\frac{1}{t^{N}}}{1-\frac{1}{t^{N}}}=\frac{\frac{a_{1}}{t}+\frac{a_{2}}{t^{2}}+\cdots+\frac{a_{N-1}}{t^{N-1}}+\frac{1}{t^{N}}}{t^{N}-1} t^{N} \\
=-\frac{a_{1} t^{N-1}+a_{2} t^{N-2}+\cdots+a_{N-1} t+1}{1-t^{N} .} .
\end{aligned}
$$

Similarly,

$$
F(f,-2 \pi y)=\frac{a_{1} t+a_{2} t^{2}+\cdots+a_{N-1} t^{N-1}+t^{N}}{1-t^{N}} .
$$

Then

$$
1+F(f,-2 \pi y)=\frac{1+a_{1} t+a_{2} t^{2}+\cdots+a_{N-1} t^{N-1}}{1-t^{N}} .
$$

Therefore, the desired limit can be written in the form

$$
\lim _{y \rightarrow+\infty} \frac{\varphi\left(x_{j}+i y\right)\left(1+a_{1} t+\cdots+a_{N-1} t^{N-1}\right)-\varphi\left(x_{j}-i y\right)\left(a_{1} t^{N-1}+\cdots+a_{N-1} t+1\right)}{1-t^{N}}
$$

where $t=e^{2 \pi y}$.

Let us represent the limit as a difference of two ones and calculate them. We obtain

$\lim _{y \rightarrow+\infty} \frac{\varphi\left(x_{j}+i y\right)\left(1+a_{1} t+\cdots+a_{N-1} t^{N-1}\right)}{1-t^{N}}$

RUSSIAN MATHEMATICS Vol.62 No.5 2018 


$$
=\lim _{y \rightarrow+\infty} \varphi\left(x_{j}+i y\right) \frac{\left(\frac{1}{e^{N 2 \pi y}}+\frac{a_{1}}{e^{(N-1) 2 \pi y}}+\cdots+\frac{a_{N-1}}{e^{2 \pi y}}\right)}{\frac{1}{e^{N 2 \pi y}}-1}=0
$$

because in the latter limit we have the product of an infinitesimal function and a bounded one $(\varphi(z)$ is bounded on the set $\left\{x_{1} \leq \operatorname{Re} z \leq x_{2}\right\}$ ).

Similarly, for the second summand we have

$$
\begin{aligned}
\lim _{y \rightarrow+\infty} \frac{\varphi\left(x_{j}-i y\right)\left(a_{1} t^{N-1}+\cdots+a_{N-1} t+1\right)}{1-t^{N}} & \\
=\lim _{y \rightarrow+\infty} \varphi\left(x_{j}-i y\right) & \frac{\left(\frac{a_{1}}{e^{2 \pi y}}+\cdots+\frac{a_{N-1}}{e^{(N-1) 2 \pi y}}+\frac{1}{e^{N 2 \pi y}}\right)}{\frac{1}{e^{N 2 \pi y}}-1}=0 .
\end{aligned}
$$

Collecting similar terms, we obtain the statement of the lemma.

Remark. From the proof of Lemma 1 it follows that

$$
\varphi\left(x_{j}-i y\right) F(f, 2 \pi y)+\varphi\left(x_{j}+i y\right)[1+F(f,-2 \pi y)], j=1,2,
$$

decreases at infinity similarly to $e^{-2 \pi y}$.

Lemma 2. We have

$$
\begin{aligned}
\lim _{y \rightarrow 0}\left(\varphi\left(x_{j}-i y\right)\right. & \left.F(f, 2 \pi y)+\varphi\left(x_{j}+i y\right)[1+F(f,-2 \pi y)]\right) \\
=\varphi\left(x_{j}\right)\left(a_{1}+\cdots+a_{N-1}\right)-\frac{2 \varphi\left(x_{j}\right)}{N}\left(a_{1}+\cdots+(N-1) a_{N-1}\right) & \quad-\frac{i \varphi_{z}^{\prime}\left(x_{j}\right)}{\pi N}\left(1+a_{1}+\cdots+a_{N-1}\right), \quad j=1,2 .
\end{aligned}
$$

Proof. We note that the functions $F(f, 2 \pi y)$ and $1+F(f,-2 \pi y)$ are defined by (12) and (13). Therefore, the desired limit can be written in the form (14) as $y \rightarrow 0$.

To find the limit of the expression of the type $\left\{\frac{0}{0}\right\}$ in (14) we will use L'Hospital's rule. Let us calculate the derivatives:

$$
\begin{gathered}
\frac{\partial}{\partial y} \varphi\left(x_{j}+i y\right)=i \varphi_{z}^{\prime}\left(x_{j}+i y\right) \\
\frac{\partial}{\partial y}\left(1+a_{1} t+\cdots+a_{N-1} t^{N-1}\right)=\left(a_{1}+\cdots+(N-1) a_{N-1} t^{N-2}\right) 2 \pi t .
\end{gathered}
$$

Therefore,

$$
\begin{aligned}
\left.\frac{\partial}{\partial y}\left[\varphi\left(x_{j}+i y\right)\left(1+a_{1} t+\cdots+a_{N-1} t^{N-1}\right)\right]\right|_{y=0}=i \varphi_{z}^{\prime}( & \left.x_{j}\right)\left(1+a_{0}+a_{1}+\cdots+a_{N-1}\right) \\
& +\varphi\left(x_{j}\right)\left(a_{1}+\cdots+(N-1) a_{N-1}\right) 2 \pi .
\end{aligned}
$$

Further,

$$
\begin{gathered}
\frac{\partial}{\partial y} \varphi\left(x_{j}-i y\right)=-i \varphi_{z}^{\prime}\left(x_{j}-i y\right) \\
\frac{\partial}{\partial y}\left(a_{1} t^{N-1}+\cdots+a_{N-1} t+1\right)=\left((N-1) a_{1}+\cdots+a_{N-1}\right) 2 \pi t .
\end{gathered}
$$

Therefore,

$$
\left.\frac{\partial}{\partial y}\left[\varphi\left(x_{j}-i y\right)\left(a_{1} t^{N-1}+\cdots+a_{N-1} t+1\right)\right]\right|_{y=0}=-i \varphi_{z}^{\prime}\left(x_{j}\right)\left(a_{0}+a_{1}+\cdots+a_{N-1}+1\right)
$$




$$
+\varphi\left(x_{j}\right)\left((N-1) a_{1}+\cdots+a_{N-1}\right) 2 \pi .
$$

We have

$$
\begin{gathered}
\frac{\partial}{\partial y}\left(1-t^{N}\right)=\frac{\partial}{\partial t}\left(1-t^{N}\right) \frac{\partial t}{\partial y}=-N t^{N-1} 2 \pi t, \\
\left.\frac{\partial}{\partial y}\left(1-t^{N}\right)\right|_{y=0}=-2 \pi N .
\end{gathered}
$$

Thus, the limit equals

$$
\begin{aligned}
& -\frac{1}{2 \pi N}\left(2 i \varphi_{z}^{\prime}\left(x_{j}\right)\left(1+a_{1}+\cdots+a_{N-1}\right)+2 \pi \varphi\left(x_{j}\right)\left(a_{1}+\cdots+(N-1) a_{N-1}\right)\right. \\
& \left.-2 \pi \varphi\left(x_{j}\right)\left((N-1) a_{1}+\cdots+[N-(N-1)] a_{N-1}\right)\right)=\varphi\left(x_{j}\right)\left(a_{1}+\cdots+a_{N-1}\right) \\
& \quad-\frac{2 \varphi\left(x_{j}\right)}{N}\left(a_{1}+\cdots+(N-1) a_{N-1}\right)-\frac{i \varphi_{z}^{\prime}\left(x_{j}\right)}{\pi N}\left(1+a_{1}+\cdots+a_{N-1}\right) .
\end{aligned}
$$

\section{THE MAIN RESULT}

Theorem 3. Let $x_{1}$ and $x_{2}$ be integers and $\varphi(z)$ be a function holomorphic and bounded on the set $\left\{x_{1} \leq \operatorname{Re} z \leq x_{2}\right\}$. Then

$$
\begin{aligned}
& \frac{P\left(w_{0}\right)}{N}\left(\frac{1}{2} \varphi\left(x_{1}\right)+\varphi\left(x_{1}+1\right)+\varphi\left(x_{1}+2\right)+\cdots+\varphi\left(x_{2}-1\right)+\frac{1}{2} \varphi\left(x_{2}\right)\right) \\
& +\frac{P\left(w_{1}\right)}{N}\left(\varphi\left(x_{1}+1-\frac{1}{N}\right)+\varphi\left(x_{1}+2-\frac{1}{N}\right)+\cdots+\varphi\left(x_{2}-1-\frac{1}{N}\right)+\varphi\left(x_{2}-\frac{1}{N}\right)\right) \\
& +\frac{P\left(w_{2}\right)}{N}\left(\varphi\left(x_{1}+1-\frac{2}{N}\right)+\varphi\left(x_{1}+2-\frac{2}{N}\right)+\cdots+\varphi\left(x_{2}-1-\frac{2}{N}\right)+\varphi\left(x_{2}-\frac{2}{N}\right)\right)+\cdots \\
& \quad+\frac{P\left(w_{N-1}\right)}{N}\left(\varphi\left(x_{1}+1-\frac{N-1}{N}\right)+\varphi\left(x_{1}+2-\frac{N-1}{N}\right)+\cdots+\varphi\left(x_{2}-1-\frac{N-1}{N}\right)\right. \\
& \left.+\varphi\left(x_{2}-\frac{N-1}{N}\right)\right)=\int_{x_{1}}^{x_{2}} \varphi(z) d z+\frac{1}{i} \int_{0}^{\infty}\left(\varphi\left(x_{1}-i y\right) F(f, 2 \pi y)+\varphi\left(x_{1}+i y\right)[1+F(f,-2 \pi y)]\right) d y \\
& \quad-\frac{1}{i} \int_{0}^{\infty}\left(\varphi\left(x_{2}-i y\right) F(f, 2 \pi y)+\varphi\left(x_{2}+i y\right)[1+F(f,-2 \pi y)]\right) d y . \quad \text { (15) }
\end{aligned}
$$

Here $F(f, 2 \pi y)=\sum_{n=1}^{\infty} e^{-q_{n} 2 \pi y}$.

Proof. We should note that $F(f, 2 \pi y)$ has a simple pole at $y=0$. For $y>0$, there are no other singularities of $F(f, 2 \pi y)$. By Lemmas 1 and 2, the integrand in (15) has singularities neither at infinity nor at $y=0$. From Remark it follows the convergence of improper integrals in the right-hand side of (15). Further we carry out our reasoning, assuming that $F(f,-2 \pi y)$ has no singularities; this is possible, because we can consider the integrals as taken over the complement of $[0, \varepsilon]$ and then proceed to the limit as $\varepsilon \rightarrow+0$.

Consider the integral

$$
\int_{\gamma_{R}} \varphi(z) F(f, 2 \pi i z) d z
$$

taken over the boundary of the rectangle $\gamma_{R}$ with vertices at the points $x_{2} \pm i R, x_{1} \pm i R, R>0$.

We note that due to singularities $x_{1}$ and $x_{2}$ of the integrand $\varphi(z) F(f, 2 \pi i z)$ lying on the contour of integration $\gamma_{R}$, the integral (16) is considered in the sense of the Cauchy principal value, i.e.,

$$
\text { v. p. } \int_{\gamma_{R}} \varphi(z) F(f, 2 \pi i z) d z \text {. }
$$


Instead of $\gamma_{R}$, we will consider the contour $\widetilde{\gamma}_{R}$, obtained from $\gamma_{R}$ by removing the segments $\left[x_{2}-i \delta, x_{2}+i \delta\right]$ and $\left[x_{1}+i \delta, x_{1}-i \delta\right]$ and replacing them by the circular arcs of radius $\delta$ centered at $\left(x_{2}, 0\right)$ and $\left(x_{1}, 0\right)$.

By Cauchy's theorem

$$
\int_{\widetilde{\gamma}_{R}} \varphi(z) F(f, 2 \pi i z) d z=\sum_{k, l} \int_{\gamma_{k, l}} \varphi(z) F(f, 2 \pi i z) d z
$$

where $\gamma_{k, l}$ is a circle of sufficiently small radius centered at $z_{k, l}$ and oriented counterclockwise. By the definition of residue,

$$
\int_{\gamma_{k, l}} \varphi(z) F(f, 2 \pi i z) d z=2 \pi i \underset{z=z_{k, l}}{\operatorname{res}}(\varphi(z) F(f, 2 \pi i z)) .
$$

Since $z_{k, l}$ are simple poles, we have

$$
\begin{gathered}
\operatorname{res}_{z=z_{k, l}}(\varphi(z) F(f, 2 \pi i z))=\operatorname{res}_{z=z_{k, l}}\left(\varphi(z) \frac{P\left(e^{-2 \pi i z}\right)}{1-e^{-2 \pi i z N}}\right)=\frac{\varphi\left(z_{k, l}\right) P\left(e^{-2 \pi i z_{k, l}}\right)}{2 \pi i N e^{-2 \pi i z_{k, l} N}} \\
=\frac{\varphi\left(z_{k, l}\right) P\left(w_{k}\right)}{2 \pi i N w_{k}^{N}}=\frac{\varphi\left(z_{k, l}\right) P\left(w_{k}\right)}{2 \pi i N} .
\end{gathered}
$$

Thus,

$$
\begin{gathered}
\int_{\gamma_{k, l}} \varphi(f, 2 \pi i z) F(f, 2 \pi i z) d z=\frac{\varphi\left(z_{k, l}\right) P\left(w_{k}\right)}{N}, \\
\int_{\widetilde{\gamma}_{R}} \varphi(z) F(f, 2 \pi i z) d z=\sum_{k, l} \varphi\left(z_{k, l}\right) \frac{P\left(w_{k}\right)}{N},
\end{gathered}
$$

where the sum is over all $z_{k, l}$ lying on $\left[x_{1}, x_{2}\right]$.

Passing to the limit as $\delta \rightarrow+0$, we obtain the left-hand side of (15). According to the residue theory, by the Privalov-Plemelj formulas, the residues at the boundary points $x_{1}$ and $x_{2}$ are taken with coefficient $1 / 2$.

On the other hand, fixing counterclockwise bypass of $\gamma_{R}$, we can represent the contour integral (16) as the sum of four integrals taken over the sides of rectangle:

$$
\begin{aligned}
& \int_{\gamma_{R}} \varphi(z) F(f, 2 \pi i z) d z \int_{x_{2}-i R}^{x_{2}+i R} \varphi(z) F(f, 2 \pi i z) d z+\int_{x_{2}+i R}^{x_{1}+i R} \varphi(z) F(f, 2 \pi i z) d z \\
& \int_{x_{1}+i R}^{x_{1}-i R} \varphi(z) F(f, 2 \pi i z) d z+\int_{x_{1}-i R}^{x_{2}-i R} \varphi(z) F(f, 2 \pi i z) d z=I_{1}+I_{2}+I_{3}+I_{4},
\end{aligned}
$$

and then we pass to the limit as $R \rightarrow+\infty$.

Now we find the value of $I_{1}$ :

$$
\begin{aligned}
& I_{1}=\int_{x_{2}-i R}^{x_{2}+i R} \varphi(z) F(f, 2 \pi i z) d z=\int_{x_{2}-i R}^{x_{2}} \varphi(z) F(f, 2 \pi i z) d z+\int_{x_{2}}^{x_{2}+i R} \varphi(z) F(f, 2 \pi i z) d z ; \\
&\left.\int_{x_{2}-i R}^{x_{2}} \varphi(z) F(f, 2 \pi i z) d z=i \int_{-R}^{0} \varphi\left(x_{2}+i y\right) F(f, 2 \pi i)\left(x_{2}+i y\right)\right) d y \\
&=-i \int_{R}^{0} \varphi\left(x_{2}-i \tau\right) F\left(f, 2 \pi i\left(x_{2}-i \tau\right)\right) d \tau=i \int_{0}^{R} \varphi\left(x_{2}-i \tau\right) F\left(f, 2 \pi i\left(x_{2}-i \tau\right)\right) d \tau
\end{aligned}
$$




$$
\begin{array}{r}
=\frac{1}{i} \int_{0}^{R}\left(-\varphi\left(x_{2}-i y\right)\right) F\left(f, 2 \pi i\left(x_{2}-i y\right)\right) d y=\frac{1}{i} \int_{0}^{R}\left(-\varphi\left(x_{2}-i y\right)\right) \sum_{n=1}^{\infty} e^{-q_{n} 2 \pi i\left(x_{2}-i y\right)} d y \\
=\frac{1}{i} \int_{0}^{R}\left(-\varphi\left(x_{2}-i y\right)\right) F(f, 2 \pi y) d y .
\end{array}
$$

Further,

$$
\begin{aligned}
\int_{x_{2}}^{x_{2}+i R} \varphi(z) F(f, 2 \pi i z) d z=i \int_{0}^{R} \varphi\left(x_{2}+i y\right) F\left(f, 2 \pi i\left(x_{2}+i y\right)\right) d y & \\
=\frac{1}{i} \int_{0}^{R} \varphi\left(x_{2}+i y\right)(-F(f,-2 \pi y)) d y & =\frac{1}{i} \int_{0}^{R} \varphi\left(x_{2}+i y\right) d y \\
& =-\frac{1}{i} \int_{0}^{R}(1+F(f,-2 \pi y)) \varphi\left(x_{2}+i y\right) d y .
\end{aligned}
$$

The equality $F\left(f, 2 \pi i\left(x_{2}+i y\right)\right)=F(f,-2 \pi y)$ holds because $e^{2 \pi i z}=e^{2 \pi i\left(x_{j}+i y\right)}=e^{-2 \pi y}, j=1,2$.

Therefore,

$$
\begin{array}{r}
I_{1}=\int_{x_{2}-i R}^{x_{2}+i R} \varphi(z) F(f, 2 \pi i z) d z=\frac{1}{i} \int_{0}^{R}\left(-\varphi\left(x_{2}-i y\right)\right) F(f, 2 \pi y) d y+\frac{1}{i} \int_{0}^{R} \varphi\left(x_{2}+i y\right) d y \\
-\frac{1}{i} \int_{0}^{R}(1+F(f,-2 \pi y)) \varphi\left(x_{2}+i y\right) d y .
\end{array}
$$

Similarly we obtain

$$
\begin{aligned}
I_{3}=\int_{x_{1}+i R}^{x_{1}-i R} \varphi(z) F(f, 2 \pi i z) d z & =-\int_{x_{1}-i R}^{x_{1}+i R} \varphi(z) F(f, 2 \pi i z) d z=\frac{1}{i} \int_{0}^{R} \varphi\left(x_{1}-i y\right) F(f, 2 \pi y) d y \\
& -\frac{1}{i} \int_{0}^{R} \varphi\left(x_{1}+i y\right) d y+\frac{1}{i} \int_{0}^{R}(1+F(f,-2 \pi y)) \varphi\left(x_{1}+i y\right) d y .
\end{aligned}
$$

We will prove for

$$
\begin{aligned}
I_{4}=\int_{x_{1}-i R}^{x_{2}-i R} \varphi(z) F(f, 2 \pi i z) d z=\int_{x_{1}}^{x_{2}} \varphi(x-i R) F(f, 2 \pi i(x-i R)) d x & \\
& =\int_{x_{1}}^{x_{2}} \varphi(x-i R) \sum_{n=1}^{\infty} e^{-q_{n} 2 \pi i x} e^{-q_{n} 2 \pi R} d x,
\end{aligned}
$$

that

$$
\begin{aligned}
\left|I_{4}\right|=\left|\int_{x_{1}}^{x_{2}} \varphi(x-i R) \sum_{n=1}^{\infty} e^{-q_{n} 2 \pi i x} e^{-q_{n} 2 \pi R} d x\right| & \leq \int_{x_{1}}^{x_{2}}|\varphi(x-i R)| \sum_{n=1}^{\infty} e^{-q_{n} 2 \pi R} d x \\
& \leq\left(x_{2}-x_{1}\right) \widetilde{C} \sum_{n=1}^{\infty} e^{-q_{n} 2 \pi R} \rightarrow 0 \text { as } R \rightarrow+\infty,
\end{aligned}
$$

since $e^{-q_{n} 2 \pi R} \rightarrow 0$ as $R \rightarrow+\infty$. We choose the constant $\widetilde{C}>0$ from the condition of boundedness of $\varphi$ on the set of integration.

To substantiate changing the order of summation and the limiting process as $R \rightarrow+\infty$, we need to 
prove uniform convergence by $R$ of the series $\sum_{n=1}^{\infty} e^{-q_{n} 2 \pi R}$ on the set $[1,+\infty)$. From (4) we have

$$
\left|\frac{1}{e^{q_{n} 2 \pi R}}\right| \leq \frac{1}{e^{q_{n} 2 \pi}} \leq \frac{1}{e^{q_{n}}}, \varlimsup_{n \rightarrow \infty} \sqrt[n]{\frac{1}{e^{q_{n}}}}=\varlimsup_{n \rightarrow \infty} \frac{1}{e^{\frac{q_{n}}{n}}}=\frac{1}{\varliminf_{n \rightarrow \infty} e^{\frac{q_{n}}{n}}}=\frac{1}{e^{\varliminf^{n \rightarrow \infty}} \frac{\lim _{n}}{n}}<1 .
$$

Therefore, the series $\sum_{n=1}^{\infty} \frac{1}{e^{q_{n}}}$ converges by Cauchy's criterion, and, by the Weierstrass $M$-test, the initial series $\sum_{n=1}^{\infty} e^{-q_{n} 2 \pi R}$ converges absolutely and uniformly by $R$ on the set $[1,+\infty)$.

Since $\varphi(z)$ is a holomorphic function on the rectangle, by Cauchy's integral theorem we obtain

$$
\int_{x_{1}}^{x_{2}} \varphi(z) d z=-\int_{x_{2}}^{x_{2}+i R} \varphi(z) d z-\int_{x_{2}+i R}^{x_{1}+i R} \varphi(z) d z-\int_{x_{1}+i R}^{x_{1}} \varphi(z) d z .
$$

Consider the integrals

$$
\begin{aligned}
& \frac{1}{i} \int_{0}^{R} \varphi\left(x_{2}+i y\right) d y-\frac{1}{i} \int_{0}^{R} \varphi\left(x_{1}+i y\right) d y+\int_{x_{2}+i R}^{x_{1}+i R} \varphi(z) F(f, 2 \pi i z) d z \\
&=-i \int_{0}^{R} \varphi\left(x_{2}+i y\right) d y-i \int_{R}^{0} \varphi\left(x_{1}+i y\right) d y+\int_{x_{2}+i R}^{x_{1}+i R} \varphi(z) F(f, 2 \pi i z) d z \\
&=\int_{x_{1}}^{x_{2}} \varphi(z) d z+\int_{x_{2}+i R}^{x_{1}+i R} \varphi(z)[1+F(f, 2 \pi i z)] d z .
\end{aligned}
$$

The final step in the proof of the theorem is to show that the integral

$$
I=\int_{x_{2}+i R}^{x_{1}+i R} \varphi(z)[1+F(f, 2 \pi i z)] d z
$$

tends to zero as $R \rightarrow+\infty$. We have

$$
I=\int_{x_{2}}^{x_{1}} \varphi(x+i R)[1+F(f, 2 \pi i(x+i R))] d x=-\int_{x_{1}}^{x_{2}} \varphi(x+i R)[1+F(f, 2 \pi i(x+i R))] d x .
$$

Since the functions $F(f, 2 \pi i z)$ and $G(w)$ are linked by the change $e^{-2 \pi i z}=w$, for $z=x+i R$ we obtain

$$
\begin{gathered}
w=e^{-2 \pi i z}=e^{-2 \pi i(x+i R)}=e^{-2 \pi i x} e^{2 \pi R}, \\
1+F(f, 2 \pi i(x+i R))=\frac{1+a_{1} w+\cdots+a_{N-1} w^{N-1}}{1-w^{N}} \\
=\frac{1+a_{1} e^{-2 \pi i x} e^{2 \pi R}+\cdots+a_{N-1} e^{-2 \pi i x(N-1)} e^{2 \pi R(N-1)}}{1-e^{-2 \pi i x N} e^{2 \pi R N}} \rightarrow 0 \text { as } R \rightarrow+\infty .
\end{gathered}
$$

Thus,

$$
\begin{aligned}
|I| \leq \int_{x_{1}}^{x_{2}}|\varphi(x+i R)| \mid 1+ & F(f, 2 \pi i(x+i R))|| d x \mid \\
\leq & \left(x_{2}-x_{1}\right) C \max _{x \in\left[x_{1}, x_{2}\right]}|1+F(f, 2 \pi i(x+i R))| \rightarrow 0 \text { as } R \rightarrow+\infty .
\end{aligned}
$$

We choose the constant $C>0$ from the condition of boundedness of the function $\varphi$ on the set of integration. Therefore, we proved that $I \rightarrow 0$ as $R \rightarrow+\infty$.

Passing to the limit in the expression for the contour integral (16) as $R \rightarrow+\infty$, we obtain 


$$
\begin{gathered}
\lim _{R \rightarrow+\infty} \int_{\gamma_{R}} \varphi(z) F(f, 2 \pi i z) d z=\lim _{R \rightarrow+\infty}\left(I_{1}+I_{2}+I_{3}+I_{4}\right) \\
=\lim _{R \rightarrow+\infty}\left(\int_{x_{2}}^{x_{2}} \varphi(z) d z+\frac{1}{i} \int_{0}^{R}\left(\varphi\left(x_{1}-i y\right) F(f, 2 \pi y)+\varphi\left(x_{1}+i y\right)[1+F(f,-2 \pi y)]\right) d y\right. \\
\left.\quad-\frac{1}{i} \int_{0}^{R}\left(\varphi\left(x_{2}-i y\right) F(f, 2 \pi y)+\varphi\left(x_{2}+i y\right)[1+F(f,-2 \pi y)]\right) d y\right) \\
=\int_{x_{1}}^{x_{2}} \varphi(z) d z+\frac{1}{i} \int_{0}^{\infty}\left(\varphi\left(x_{1}-i y\right) F(f, 2 \pi y)+\varphi\left(x_{1}+i y\right)[1+F(f,-2 \pi y)]\right) d y \\
\quad-\frac{1}{i} \int_{0}^{\infty}\left(\varphi\left(x_{2}-i y\right) F(f, 2 \pi y)+\varphi\left(x_{2}+i y\right)[1+F(f,-2 \pi y)]\right) d y .
\end{gathered}
$$

The latter equality follows from Remark.

In the case $q_{n}=n$, we have $G(w)=\frac{w}{1-w}, P(1)=1$. From (12), (13), and reasoning on rationality of the function $F(f,-2 \pi y)$, given in the proof of Lemma 1, we deduce

$$
\begin{gathered}
F(f, 2 \pi y)=\sum_{n=1}^{\infty} e^{-n 2 \pi y}=e^{-2 \pi y}+e^{-4 \pi y}+e^{-6 \pi y}+\cdots=\frac{e^{-2 \pi y}}{1-e^{-2 \pi y}}=\frac{1}{e^{2 \pi y}-1}, \\
1+F(f,-2 \pi y)=1+\frac{w}{1-w}=\frac{1}{1-w}=\frac{1}{1-e^{2 \pi y}}=-\frac{1}{e^{2 \pi y}-1},
\end{gathered}
$$

and, consequently, we obtain the Plana formula (1).

The proven generalization of the Plana formula can be further used in obtaining an analog of Binet's integral representation. In that way, the transition will be implemented to obtaining the functional equation for the zeta-function of zeroes for some class of entire functions.

\section{ACKNOWLEDGMENTS}

Supported by the Russian Foundation for Basic Research (projects No. 15-01-00277, 16-31-00173) and the grant of the Government of the Russian Federation for research under supervision of leading scientists in Siberian Federal University (contract No. 14.Y26.31.0006).

\section{REFERENCES}

1. Whittaker, E. T., Watson, G. N. A Course of Modern Analysis (Cambridge University Press, Cambridge, 1927).

2. Titchmarsh, E. C. The Theory of the Riemann Zeta-Function (Oxford University Press, Oxford, 1951).

3. Kuzovatov, V. I., Kytmanov, A. M. "On an Analog of the Plana Formula", NAN Armenii. Matem. 53, No. 3, $1-12$ (2018) [in Russian].

4. Gel'fand, I. M., Levitan, B. M. "On a Simple Identity for Eigenvalues of a Second Order Differential Operator”, Sov. Phys. Dokl. 88, No. 4, 593-596 (1953) [in Russian].

5. Dikii, L. A. "The Zeta-Function of an Ordinary Differential Equation on a Finite Interval", Izv. Akad. Nauk SSSR Ser. Mat. 19, No. 4, 187-200 (1955) [in Russian].

6. Dikii, L. A. "Trace Formulas for Sturm-Liouville Differential Operators", Uspekhi Mat. Nauk 13, No. 3, 111-143 (1958) [in Russian],

7. Lidskii, V. B., Sadovnichii, V. A. "Regularized Sums of Zeros of a Class of Entire Functions", Functional Analysis and its Applications 1, No. 2, 133-139 (1967).

8. Smagin, S. A., Shubin, M. A. "On the Zeta-Function of a Transversally Elliptic Operator", Russian Mathematical Surveys 39, No. 2, 201-202 (1984).

9. Kytmanov, A. M., Myslivets, S. G. "On the Zeta-Function of Systems of Nonlinear Equations", Siberian Math. J. 48, No. 5, 863-870 (2007).

10. Kuzovatov, V. I., Kytmanov, A. A. "On the Zeta-Function of Zeros of Some Class of Entire Functions", J. Siberian Federal Univ. Math. Phys. 7, No. 4, 489-499 (2014).

11. Bieberbach, L. Analytische Fortsetzung (Springer-Verlag, Berlin, 1955).

Translated by S. R. Nasyrov 